\title{
Modelos forzados y no forzados para el cálculo del índice de sitio en bosques de Nothofagus antarctica en Patagonia Sur
}

\author{
Constrained and non-constrained models for site index estimation in Nothofagus antarctica \\ forests of South Patagonia
}

\author{
Horacio Ivancich $^{\text {a*}}$, Guillermo Martínez Pastur ${ }^{\text {a }}$, Pablo Luis Peri ${ }^{\mathrm{b}}$ \\ *Autor de correspondencia: ${ }^{a}$ Centro Austral de Investigaciones Científicas (CADIC CONICET), Houssay 200 (9410) Ushuaia, \\ Tierra del Fuego, Argentina, tel.: +54-2901-422310, fax: +54-2901-430644, horacioivancich@cadic-conicet.gob.ar \\ ${ }^{\mathrm{b}}$ UNPA INTA CONICET, Río Gallegos, Santa Cruz, Argentina.
}

\begin{abstract}
SUMMARY
Yield potential of a stand can be defined through site quality. One way to measure site quality is using site index equations, being available different equation types (constrained and non-constrained). The objective of this study was to compare the fitness of site index equations using different base ages, and validate them along site quality and age classes in Nothofagus antarctica forests of South Patagonia. Four height growth equations were fitted using two constrained and two non-constrained models. The equations were fitted using three base ages (30, 40 and 50 years). Self-validation was done using gradients of age and site quality. Lower errors were obtained when a base age of 50 years was used for all the equations. Besides this, the best fitting along the age and site quality gradients were obtained with Weibull, Richards and Newnham equations. The self-validation values of the selected equation were comparable to the fitting presented by other authors, who worked with site index models in Nothofagus spp. forests. Newnham equation better described the height dominant growth in $N$. antarctica, which presented great biological significance according to the shape of the obtained curves. Hence, this model can be recommended to be used as a prediction tool for forest management planning in $N$. antarctica forests.
\end{abstract}

Key words: site index, stem analysis, constrained models; non-constrained models.

\section{RESUMEN}

El potencial de producción de un rodal puede definirse a través de la calidad de sitio. Una forma de medir dicha calidad es a través de ecuaciones de índice de sitio, existiendo distintos tipos de ecuaciones (forzadas y no forzadas). El objetivo fue comparar el ajuste de diferentes ecuaciones de índice de sitio con diferentes edades base mediante validaciones a lo largo de gradientes de edad y clase de sitio en bosques de Nothofagus antarctica de Patagonia Sur. Se ajustaron cuatro ecuaciones de crecimiento en altura, dos no forzadas y dos forzadas. Las edades base utilizadas para el ajuste fueron 30, 40 y 50 años. Se realizaron autovalidaciones de las ecuaciones a lo largo de gradientes de edad y de calidad de sitio. Los menores errores se obtuvieron al utilizar una edad base de 50 años para todas las ecuaciones. Los mejores ajustes en los gradientes de edad y calidad de sitio se obtuvieron con los modelos de Weibull, Richards y Newnham. Los valores provenientes de la autovalidación de las ecuaciones seleccionadas fueron comparables a los encontrados por otros autores que trabajaron con curvas de índice de sitio aplicadas a bosques de Nothofagus spp. La ecuación de Newnham fue la que mejor describió el crecimiento en altura dominante de $N$. antarctica, cumpliendo con las condiciones que deben tener las curvas de crecimiento. Estos resultados permiten señalar que el modelo es recomendable para ser usado como una herramienta de predicción en la planificación del manejo de los bosques de $N$. antarctica.

Palabras clave: índice de sitio, análisis fustal, modelos forzados, modelos no forzados.

\section{INTRODUCCIÓN}

Para una correcta planificación del manejo forestal es necesario contar con herramientas precisas de predicción. Los modelos dendrométricos relacionados con la productividad son eficaces para definir el potencial de un sitio, analizar el funcionamiento de los ecosistemas forestales (McDill y Amateis 1992) y para planificar el manejo forestal (Gregoire 1993). El potencial de producción de un rodal puede definirse a través de la calidad de sitio, el cual depende de la combinación de factores físicos y biológicos de una localiza- ción geográfica particular (Skovsgaard y Vanclay 2008). En el caso de los bosques de Nothofagus antarctica (Forster f.) Oersted, comúnmente llamado 'ñire' o 'ñirre', la calidad de sitio se encuentra en estrecha relación con una combinación de factores del ambiente: suelo (profundidad y nutrientes), régimen hídrico (pendiente, exposición, evapotranspiración) y temperatura, entre otros. Debido a esto, los bosques de ñire pueden alcanzar alturas dominantes de cuatro a 18 metros. Una forma indirecta de medir la calidad de sitio es a través del desarrollo de ecuaciones de índice de sitio (IS) (Monserud 1984), definido como la altura promedio de los 
árboles dominantes de un rodal no disturbado, coetáneo y adecuadamente denso a una edad base o de referencia (Thrower 1989). Dicho índice se basa sobre dos supuestos: (i) que el crecimiento en altura depende en gran medida de la calidad de sitio, y que es independiente de la densidad; y (ii) que el crecimiento en altura es asintótico a lo largo del tiempo alcanzando un máximo que es definido por la calidad de sitio (Sturtevant y Seagle 2004).

Las ecuaciones de índice de sitio pueden ser de tipo anamórfico o polimórfico. En los sistemas de curvas anamórficas, la forma de las curvas de crecimiento en altura son iguales para todas las calidades de sitio. Esta condición no está impuesta en los sistemas de curvas polimórficas donde la forma de las curvas de crecimiento de la altura varía de acuerdo con la edad para cada calidad de sitio en particular (Trincado et al. 2002). Generalmente en los estudios de crecimiento biológico, y específicamente en aquellos utilizados para la construcción de curvas de índice de sitio, se recomiendan aquellas ecuaciones que denoten curvas polimórficas en su estructura (García 1983), debido a que estas se elaboran sin considerar una constante al parámetro de la asíntota (Esse et al. 2007). A su vez, las ecuaciones polimórficas pueden dividirse en forzadas o no forzadas de acuerdo a las variables de ajuste que incluye en su composición (Payandeh y Wang 1994, Wang y Payandeh 1994a). Las ecuaciones forzadas producen curvas que predicen una altura a la edad base coincidente al índice de sitio, mientras que las ecuaciones no forzadas predicen alturas a la edad base que difieren del índice de sitio.

Otra característica a considerar en la construcción de los modelos es la elección de la edad base. La edad base es elegida arbitrariamente y no posee una significancia biológica especial (Thrower 1989), pero influye en el ajuste de los modelos. En bosques templados suelen utilizarse edades base de 25 a 100 años (Thrower y Goudie 1992, Martínez Pastur et al. 1997, Splechtna 2001, Sturtevant y Seagle 2004, Upadhyay et al. 2005). El concepto de índice de sitio ha sido desarrollado principalmente para ser aplicado en plantaciones coetáneas monoespecíficas (Clutter et al. 1983). Sin embargo, la dinámica simple de los bosques nativos del género Nothofagus (Donoso 2006) que forman bosques puros y dan origen a rodales homogéneos permite que el concepto de índice de sitio sea utilizado en estos bosques (Martínez Pastur et al. 1997, Trincado et al. 2002, Salas y García 2006, Esse et al. 2007). Se han desarrollado ecuaciones para bosques de Nothofagus pumilio (Poepp. et Endel.) Krasser, N. dombeyi (Mirb.) Oerst., N. obliqua (Mirb.) Oerst. y N. alpina Bird. Egefam., tanto anamórficas como polimórficas así como del tipo forzado y no forzado empleando edades base de 20 a 60 años. Para el caso particular de $N$. antarctica, solo se publicó una clasificación preliminar de calidades de sitio a partir de la altura dominante de rodales en fases de crecimiento avanzadas (Lencinas et al. 2002), sin haberse desarrollado ecuaciones de índice de sitio. En dicha clasificación se estableció un rango de alturas dominantes para rodales adultos que va desde alturas menores a $6 \mathrm{~m}$ hasta más de $12 \mathrm{~m}$. El uso de modelos de índice de sitio apropiados para la especie mejoran los cálculos de posibilidad de cosecha (Thrower 1989), así como también permiten obtener estimaciones de volumen mas precisas que constituyen información indispensable para la planificación de un correcto manejo forestal. Pero no existe en la bibliografía una clara discusión de cómo influyen los distintos modelos y parámetros en el ajuste de este tipo de ecuaciones. Debido a esto, la evaluación de este tipo de modelos, para la cual existe escasa o nula información, brindará información valiosa a los administradores de este recurso forestal. Se considera como hipótesis de trabajo que el empleo de distinto tipo de modelos y diferentes parámetros de base para el desarrollo de ecuaciones de índice de sitio influyen de manera considerable en la calidad de ajuste de los mismos. Es por ello que el objetivo de este trabajo es comparar el ajuste de ecuaciones de índice de sitio forzadas y no forzadas con diferentes edades base mediante validaciones a lo largo de gradientes de edades y clases de sitio en bosques de $N$. antarctica de Patagonia Sur.

\section{MÉTODOS}

Se muestrearon 72 árboles dominantes en bosques primarios y secundarios de ñire en Tierra del Fuego y Santa Cruz, Argentina (estancia Los Cerros 54 22' $18^{\prime \prime} \mathrm{S}, 67^{\circ}$ $51^{\prime} 08^{\prime \prime} \mathrm{O}$; estancia Stag River $51^{\circ} 38^{\prime} 60^{\circ}$ ' S, $72^{\circ} 01^{\prime} 00^{\prime \prime}$ O y estancia La Suerte $49^{\circ} 08^{\prime} 49^{\prime \prime}$ S, $72^{\circ} 58^{\prime} 03^{\prime \prime}$ O). El clima en esta región es, por lo general, moderado como consecuencia de las pequeñas masas continentales en proporción a las grandes masas oceánicas. La precipitación, que varía entre 400 y $800 \mathrm{~mm}$ año ${ }^{-1}$, se distribuye en forma regular durante todo el año. Los suelos, en general de textura franco-arenosa, presentan como componente principal a la morena o material de arrastre de los glaciares (Tuhkanen 1989-1990). Tienen abundante materia orgánica y nitrógeno en superficie, pero están lavados de sales solubles y están casi desprovistos de fósforo.

Los rodales muestreados fueron puros, coetáneos y de densidad completa. Los muestreos se limitaron a los bosques de morfotipo arborescente (Donoso 2006), excluyendo el resto de los morfotipos (arbustivo o achaparrado, caméfito o postrado) propios de ambientes más rigurosos. Se seleccionaron árboles dominantes con buena sanidad externa y sin daños evidentes en la copa o el fuste, los que fueron apeados para la toma de las muestras. Del fuste principal de cada individuo se extrajeron rodajas a los $1,3 \mathrm{~m}, 2,0 \mathrm{~m}$ y a intervalos de un metro hasta alcanzar la altura total del individuo. En dichas rodajas se realizó la lectura de anillos para la obtención de pares de datos altura-edad. A la base de datos obtenida se le adicionaron 47 mediciones puntuales de individuos dominantes a los que se les determinó la edad a partir de mediciones de barreno. Se obtuvo un total de 653 pares de datos altura-edad (cuadro 1). 
Cuadro 1. Conjunto de datos para la modelación del crecimiento en altura de $N$. antarctica.

Final data set for height growth modeling of $N$. antarctica.

\begin{tabular}{lcc}
\hline Parámetro & Edad (años) & Altura $(\mathrm{m})$ \\
\hline Promedio & 62,2 & 7,2 \\
Máximo & 259 & 15,8 \\
Mínimo & 0 & 1,3 \\
Desvío estándar & 51,9 & 3,3 \\
$\mathrm{n}$ & 653 & 653 \\
\hline
\end{tabular}

A cada árbol se le calculó un índice de sitio para las tres edades bases ensayadas (30, 40 y 50 años) mediante interpolaciones entre las mediciones sucesivas. Se consideró como edad cero a la medida a 1,3 m del suelo, de modo de disminuir los efectos de factores externos a la calidad de sitio que puedan influir sobre el crecimiento en altura de los árboles en edades tempranas (Thrower 1989) como, por ejemplo, competencia, ramoneo de guanaco (Lama guanicoe, Camelidae), liebre doméstica (Lepus europeaus, Lagomorpha) o ganado doméstico (Martínez Pastur et al. 1999, Pulido et al. 2000). Esta misma metodología fue utilizada por otros autores para bosques de Pseudotsuga menziesii (Mirb.) Franco y Pinus ponderosa (Dougl.) Laws (Monserud 1984, Waring et al. 2006) de modo de disminuir la variabilidad asociada al periodo inicial de establecimiento.

Con los datos obtenidos se ajustaron cuatro funciones de crecimiento en altura $(H)$, donde las variables independientes fueron la edad de los árboles a 1,3 m (t) y el índice de sitio $\left(\mathrm{IS}_{\mathrm{ti}}\right)$ a una edad base determinada (ti), de acuerdo a datos requeridos por las ecuaciones empleadas. Se analizaron dos ecuaciones no forzadas de cinco parámetros: la función de crecimiento de Weibull propuesta por Zeide (1993) [1] y una función basada en la función biológica de crecimiento de Richards [2] (Ek 1971, Wang y Payandeh 1994b). Asimismo se escogieron dos ecuaciones forzadas, una de cuatro parámetros propuesta por Goelz y Burk (1992) [3] y una de tres parámetros propuesta por Newnham (1988) [4]. Para el ajuste de los datos, a la variable $\mathrm{IS}_{\mathrm{ti}}$ se le restó 1,3 m. Esta modificación, empleada por Martínez Pastur et al. (1997) para Nothofagus pumilio, es debida a que se considera la edad total del árbol a 1,3 metros de altura.

$$
H=\left(a\left(I S_{t i}-1,3\right)^{b}\left(1-e^{c t^{\left(d\left(I S_{i i}-1,3\right)^{e}\right)}}\right)+1,3\right.
$$

$H=\left(a\left(I S_{t i}-1,3\right)^{b}\left(1-e^{c t}\right)^{\left(d\left(I S_{t i}-1,3\right)^{e}\right)}\right)+1,3$

$$
\begin{aligned}
& H=\left(\left(I S_{t i}-1,3\right)\left(\frac{1-\exp \left(-a\left(\frac{I S_{t i}}{t i}\right)^{b} t i^{c} t\right)}{1-\exp \left(-a\left(\frac{I S_{t i}}{t i}\right)^{b} t i^{c} t i\right)}\right)^{d}+1,3\right. \\
& H=\left(a\left(I S_{t i}-1,3\right)^{b}\left(1-e^{-c t}\right)^{f}\right)+1,3 \\
& f=\frac{\ln \left(\frac{\left(I S_{t i}-1,3\right)^{1-b}}{a}\right)}{\ln \left(1-e^{-c t i}\right)}
\end{aligned}
$$

Donde H: altura total (m), IS: índice de sitio (m), t: edad (años), ti: edad base (años), ln: logaritmo natural, $e$ : base de los logaritmos neperianos, a-b-c-d-e: parámetros de los modelos. [1]: Weibull, [2]: Richards [3]: Goelz y Burk, [4]: Newnham.

Las ecuaciones elegidas fueron ajustadas para las tres edades base propuestas ( 30,40 y 50 años) obteniendo en total 12 ecuaciones de crecimiento en altura. Todas las ecuaciones se ajustaron mediante técnicas de regresión no lineal (Gezan et al., 2009) utilizando el algoritmo de Marquardt. En los últimos años se han publicado diversos trabajos donde se describen las bondades de de este tipo de metodologías (modelos no lineales y algoritmo de Marquardt) (e.g. Khamis et al. 2005, Lei y Zhang 2004, Fekedulegn et al. 1999). La evaluación estadística de los modelos se llevó a cabo a través del coeficiente de determinación ajustado ( $\mathrm{R}^{2}$-ajust.) y se evaluó la correlación de errores a partir del coeficiente de Durbin-Watson. También se realizó un análisis de los residuales a partir del error medio de la estimación (EME), el error estándar de la estimación (ESE), el error medio absoluto (EMA), el error medio porcentual $(\% \overline{\mathrm{e}})$ y el error medio porcentual en valor absoluto (\%|ē|) de acuerdo a las siguientes ecuaciones:

$$
\begin{aligned}
& \left.\mathrm{EME}=\left(\sum_{i=1}^{n} e_{i}\right) / n\right) \\
& \left.\mathrm{ESE}=\left(\sum_{i=1}^{n} e_{i}\right)^{2} / n\right) \\
& \left.\mathrm{EMA}=\left(\sum_{i=1}^{n}\left|e_{i}\right|\right) / n\right) \\
& \left.\% \overline{\mathrm{e}}=\left(\sum_{i=1}^{n} e_{i} / x_{i}\right) / n\right) \\
& \left.\%|\overline{\mathrm{e}}|=\left(\sum_{i=1}^{n}\left|e_{i} / x_{i}\right|\right) / n\right)
\end{aligned}
$$

Donde $n$ es el número de datos utilizados en el ajuste del modelo, $e_{i}$ el residuo (valor observado - valor predicho) y $x_{i}$ la altura observada. 
La edad de referencia se definió a partir del mejor ajuste estadístico para cada ecuación, basado en los estadísticos de las ecuaciones y el análisis de los residuales. Una vez seleccionada una edad base se realizó una autovalidación de los ecuaciones ajustados en la que se analizó la distribución porcentual del error medio absoluto, el error medio de la estimación y el error medio porcentual en valor absoluto a lo largo de un gradiente de clases de edades $(<49$; 50-99; 100-149; > 150 años) y clases de sitio, las que fueron definidas para cada ecuación, según el IS a la edad base seleccionada. Así mismo, se compararon mediante análisis de varianza $(P<0,05)$ el error medio de la estimación y el error medio porcentual en valor absoluto entre ecuaciones para cada clase de sitio y de edad. Los límites de cada intervalo de índice de sitio se fijaron dividiendo su rango total en cuatro clases (I, II, III y IV, siendo I la mejor y IV la peor). A partir de la base de datos obtenida y de las ecuaciones de crecimiento en altura dominante ajustados, se conformó una nueva clasificación de calidades de sitio según el índice de sitio a la edad base seleccionada.

\section{RESULTADOS}

Las ecuaciones no forzadas explicaron entre el $89 \%$ y el $94 \%$ de la variación de los datos, muy similar a las ecuaciones forzadas que explicaron entre el $88 \%$ y el 94 $\%$ de la variación (cuadro 2). Los mayores valores del coeficiente de determinación ajustado ( $\mathrm{R}^{2}$-ajust.) de cada ecuación se obtuvieron con la mayor edad base ensayada
(50 años), mientras que los peores ajustes se obtuvieron con una edad base de 30 años. La ecuación no forzada [2] fue la que presentó los mayores valores de $\mathrm{R}^{2}$-ajust. para edades base de 30 y 50 años, mientras que los menores ajustes se obtuvieron con la ecuación [4] a una edad base de 30 años. Comparativamente, entre las dos ecuaciones forzadas, la ecuación de cuatro parámetros [3] presentó coeficientes de determinación levemente mayores que la ecuación de tres parámetros [4] para todas las edades de referencia ensayadas.

No se detectaron grandes diferencias entre ecuaciones no forzadas y forzadas al analizar la autocorrelación de errores, respecto al coeficiente de Durbin-Watson (cuadro 3). Por otra parte, la mayor autocorrelación se observó cuando se utilizó la edad base de 50 años para todas las ecuaciones.

En general los errores globales presentaron menores valores para las ecuaciones no forzadas en comparación con las ecuaciones forzadas (cuadro 4). El error medio de la estimación varió entre $-0,01 \mathrm{~m}$ y $0,03 \mathrm{~m}$ para las ecuaciones no forzadas, y entre $-0,20 \mathrm{~m}$ y $0,08 \mathrm{~m}$ para las ecuaciones forzadas. El error estándar de la estimación varió de $0,88 \mathrm{~m}$ a $1,07 \mathrm{~m}$, y de $0,89 \mathrm{~m}$ a $1,09 \mathrm{~m}$ para las ecuaciones no forzadas y forzadas, respectivamente. El error medio absoluto varió de $0,66 \mathrm{~m}$ a $0,77 \mathrm{~m}$, y de $0,63 \mathrm{~m}$ a $0,73 \mathrm{~m}$ para las ecuaciones no forzadas y forzadas, respectivamente. Los errores medios porcentuales $(\% \overline{\mathrm{e}})$ variaron de $1,8 \%$ a $4,7 \%$, y de $5,3 \%$ a $10,3 \%$ para las ecuaciones no forzadas y forzadas, respectivamente. Y los errores medios porcentuales absolutos $(\%|\bar{e}|)$ variaron de $12,4 \%$

Cuadro 2. Parámetros y estadísticos de los modelos de índice se sitio ajustados para N. antarctica. Parameters and statistics of the site index models for $N$. antarctica.

\begin{tabular}{ccccccccc}
\hline Modelos & Ecuación & $\begin{array}{c}\text { Edad base } \\
\text { (años) }\end{array}$ & $\mathrm{a}$ & $\mathrm{b}$ & $\mathrm{c}$ & $\mathrm{d}$ & $\mathrm{e}$ & $\mathrm{R}^{2}$-ajust. \\
\hline \multirow{6}{*}{ No } & {$[1]$} & 30 & 7,3470 & 0,2694 & $-0,0139$ & 0,8648 & 0,1465 & 89,14 \\
forzados & {$[1]$} & 40 & 6,9284 & 0,2853 & $-0,0111$ & 0,7954 & 0,2036 & 92,27 \\
& {$[1]$} & 50 & 7,0429 & 0,2823 & $-0,0112$ & 0,8126 & 0,1642 & 94,06 \\
& {$[2]$} & 30 & 6,1211 & 0,3865 & $-0,0211$ & 2,3749 & $-0,4708$ & 89,59 \\
& {$[2]$} & 40 & 5,0663 & 0,4693 & $-0,0206$ & 3,4972 & $-0,6156$ & 92,07 \\
\multirow{5}{*}{ Forzados } & {$[2]$} & 50 & 5,5198 & 0,4222 & $-0,0181$ & 3,6200 & $-0,6069$ & 94,36 \\
\hline & {$[3]$} & 30 & 0,3965 & 0,8437 & $-0,3434$ & 1,3367 & - & 89,42 \\
& {$[3]$} & 40 & 0,2089 & 0,8623 & $-0,1614$ & 1,2486 & - & 92,56 \\
& {$[3]$} & 50 & 0,2902 & 0,9936 & $-0,1643$ & 1,3100 & - & 94,11 \\
& {$[4]$} & 30 & 5,9613 & 0,3899 & $-0,0243$ & - & - & 88,14 \\
& {$[4]$} & 40 & 4,7707 & 0,4986 & $-0,2253$ & - & - & 91,84 \\
& {$[4]$} & 50 & 5,3209 & 0,4428 & $-0,0197$ & - & - & 93,93 \\
\hline
\end{tabular}

[1]: modelo de Weibull; [2]: modelo de Richards; [3]: modelo forzado de Goelz-Burk; [4]: modelo forzado de Newnham; a-b-c-d-e: parámetros de la ecuación; $\mathrm{R}^{2}$-ajust.: coeficiente de determinación ajustado; -: sin parámetro.

[1]: Weibull model; [2]: Richards model; [3]: Goelz-Burk constrained model; [4]: Newnham constrained model; a-b-c-d-e: equation parameters; $\mathrm{R}^{2}-$ ajust.: adjusted coefficient of determination; -: no parameter. 
Cuadro 3. Valores de los coeficientes de Durbin-Watson para los modelos ajustados.

Coefficient values of Durbin-Watson of fitted models.

\begin{tabular}{cccccc}
\hline \multirow{2}{*}{ Modelos } & \multirow{2}{*}{ Ecuación } & \multicolumn{3}{c}{ Edad base (años) } & \multirow{2}{*}{ Promedio } \\
\cline { 3 - 6 } & & 30 & 40 & 50 & \\
\hline \multirow{2}{*}{$\begin{array}{c}\text { No } \\
\text { forzados }\end{array}$} & {$[1]$} & 1,6537 & 1,6235 & 0,6342 & 1,3038 \\
& {$[2]$} & 1,6226 & 1,5838 & 0,7842 & 1,3302 \\
\hline \multirow{2}{*}{ Forzados } & {$[3]$} & 1,7136 & 1,6437 & 0,6637 & 1,3403 \\
& {$[4]$} & 1,6017 & 1,5476 & 0,7752 & 1,3082 \\
\hline Promedio & & 1,6479 & 1,5997 & 0,7143 & - \\
\hline
\end{tabular}

a $15,3 \%$, y de $12,9 \%$ a $17,0 \%$ para las ecuaciones no forzadas y forzadas, respectivamente. Por otra parte, los menores errores se obtuvieron cuando se utilizó una edad base de 50 años para todas las ecuaciones.

En la autovalidación de las ecuaciones, el rango de índice de sitio para una edad base de 50 años $\left(\mathrm{IS}_{50}\right)$ que-

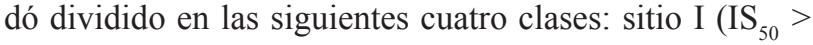
9,0 m), sitio II ( $\operatorname{IS}_{50} 7,3 \mathrm{~m}$ a 9,0 m), sitio III ( $\operatorname{IS}_{50} 5,5 \mathrm{~m}$ a 7,2 $\mathrm{m})$ y sitio IV ( $\operatorname{IS}_{50}<5,5 \mathrm{~m}$ ). Considerando una edad base de 50 años, las distribuciones del error medio absoluto por clase de sitio y clase de edad fueron equilibradas para todas ecuaciones consideradas (figura 1) ya que en ningún caso la diferencia de error medio absoluto entre la mayor y la menor clase de edad fue superior al $14 \%$ mientras que la diferencia entre la menor y mayor clase de sitio no superó el $13 \%$. Por otro lado, no se manifestó una diferenciación evidente entre ecuaciones forzadas y no forzadas. Los menores valores de error medio absoluto se obtuvieron en la clase inferior de edad para todos los modelos (18\% a $22 \%$ ) (figura $1 \mathrm{~A})$. Estos errores fueron aumentando con la edad, llegando a valores de $29 \%$ a $32 \%$ en la clase superior (> 150 años). El modelo [1] fue el que pre- sentó la mayor variabilidad del error medio absoluto entre clases de edad con diferencias porcentuales entre la mayor y menor clase del $14 \%$, y el modelo [4] fue el que presentó la menor diferencia $(7 \%)$. Los porcentajes de error medio absoluto por clases de sitio presentaron un aumento indirectamente proporcional con la clase considerada para todos los modelos analizados (figura 1B). Las diferencias de porcentaje de error medio absoluto entre la clase de sitio superior e inferior para las ecuaciones no forzadas fueron del $12 \%$, mientras que para las ecuaciones forzadas dicha diferencia varió entre el $10 \%$ y el $13 \%$.

Para la mejor clase de sitio (I) las ecuaciones [2] y [4] presentaron un error medio de la estimación significativamente superiores $(P<0,05)$ al resto de las ecuaciones (figura 2A). En las clases de sitio intermedias (II y III) las ecuaciones no forzadas presentaron valores sobreestimados, mientras que las ecuaciones forzadas subestimaron las alturas en ambas clases de sitio. En la clase de sitio IV las ecuaciones forzadas [3] y [4] subestimaron diferenciándose significativamente de la ecuación no forzada [4], mientras que la ecuación [2] subestimo levemente sin diferenciarse significativamente del resto de las ecuaciones. $\mathrm{Al}$ analizar la distribución del error medio de la estimación por clases de edades (figura 2B), los valores fueron positivos en las primeras tres clases de edades $(<150$ años $)$ para las ecuaciones no forzadas, y negativos para la clase de edad superior (> 150 años). Por otro lado, las ecuaciones forzadas tuvieron un patrón de distribución de errores diferente de las ecuaciones no forzadas, donde el modelo [3] subestimó en todas las clases de edades, y donde la ecuación de Newnham [4] sobreestimó en las clases de edad intermedias y subestimó en el resto de las clases $(<50$ años $\mathrm{y}>150$ años $)$.

Los errores medios porcentuales en valor absoluto $(\%|\bar{e}|)$ no se diferenciaron significativamente entre ecua-

Cuadro 4. Errores medios de los modelos de índice se sitio ajustados para N. antarctica. Mean errors of site index models for $N$. antarctica.

\begin{tabular}{|c|c|c|c|c|c|c|c|}
\hline Modelos & Ecuación & Edad base (años) & $\operatorname{EME}(\mathrm{m})$ & $\operatorname{ESE}(\mathrm{m})$ & $\operatorname{EMA}(\mathrm{m})$ & $\% \overline{\mathrm{e}}(\%)$ & $\%|\bar{e}|(\%)$ \\
\hline \multirow{6}{*}{$\begin{array}{c}\text { No } \\
\text { forzados }\end{array}$} & {$[1]$} & 30 & $<0,01$ & 1,07 & 0,77 & 4,71 & 15,28 \\
\hline & [1] & 40 & $-0,01$ & 0,92 & 0,66 & 1,96 & 15,02 \\
\hline & [1] & 50 & $-0,02$ & 0,91 & 0,68 & 1,82 & 13,66 \\
\hline & [2] & 30 & $-0,01$ & 1,07 & 0,76 & 3,54 & 12,83 \\
\hline & [2] & 40 & $-0,02$ & 0,93 & 0,67 & 2,27 & 12,54 \\
\hline & [2] & 50 & $-0,03$ & 0,88 & 0,67 & 2,55 & 12,42 \\
\hline \multirow{6}{*}{ Forzados } & [3] & 30 & $-0,01$ & 1,07 & 0,73 & 8,93 & 16,90 \\
\hline & [3] & 40 & $-0,07$ & 0,89 & 0,63 & 10,26 & 17,03 \\
\hline & [3] & 50 & $-0,20$ & 0,87 & 0,67 & 9,27 & 15,81 \\
\hline & [4] & 30 & 0,08 & 1,09 & 0,76 & 6,05 & 13,61 \\
\hline & {$[4]$} & 40 & $<0,01$ & 0,95 & 0,68 & 6,51 & 12,91 \\
\hline & [4] & 50 & $-0,06$ & 0,92 & 0,70 & 5,33 & 13,22 \\
\hline
\end{tabular}

[1]: modelo de Weibull; [2]: modelo de Richards; [3]: modelo forzado Goelz-Burk; [4]: modelo forzado de Newnham; EME: error medio de la estimación; ESE: error estándar de la estimación; EMA: error medio absoluto; \%ē: error medio porcentual; \%|ê||: error medio porcentual en valor absoluto.

[1]: Weibull model; [2]: Richards model; [3]: Goelz-Burk constrained model; [4]: Newnham constrained model; EME: mean error of estimate; ESE: standard error of estimate; EMA: mean absolute error; \% $\%$ : mean percentage error; \%| $\mid$ |: mean absolute percentage error. 


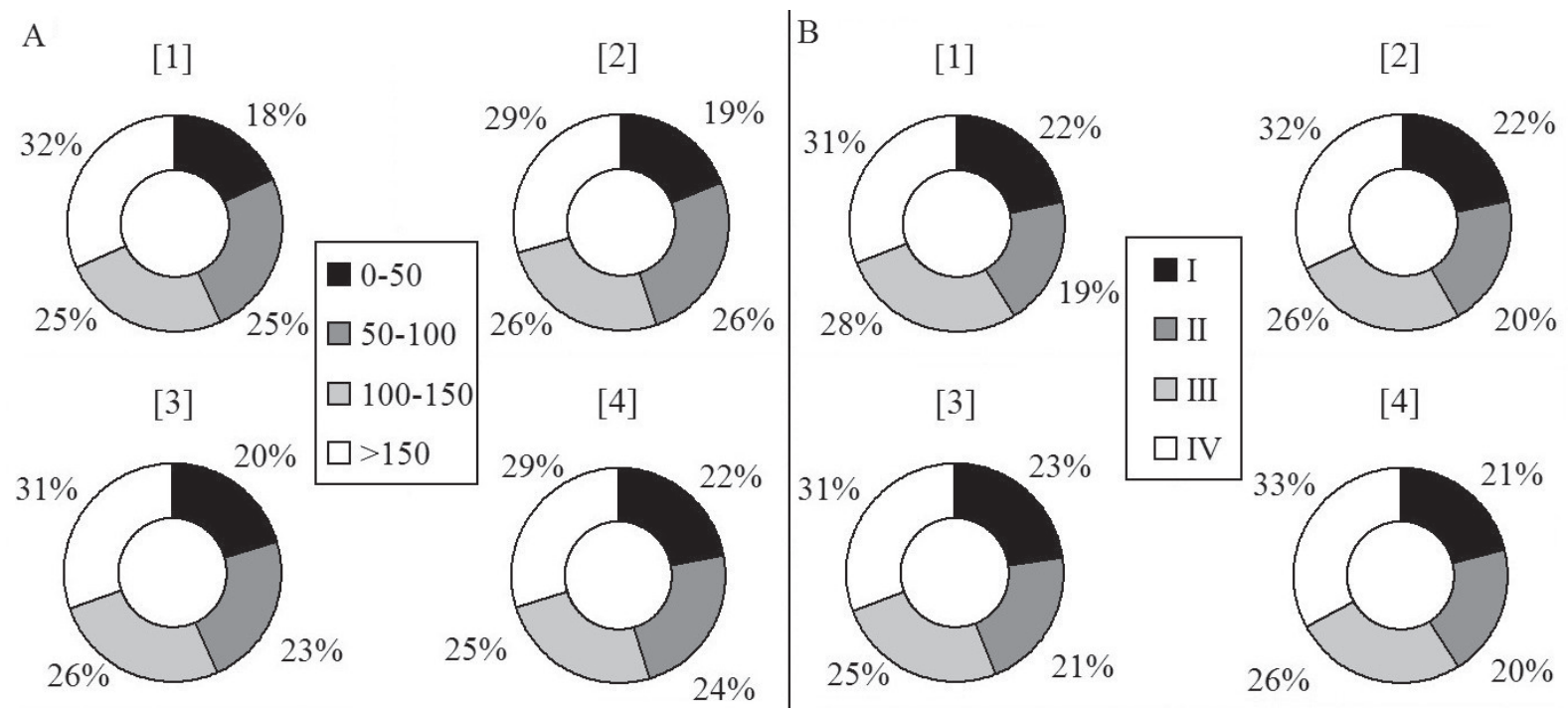

Figura 1. Comparación de los errores globales absolutos por clase de sitio (A) y por clase de edad (B) utilizando una edad base de 50 años.

Comparison of absolute global errors by site (A) and age classes (B) using a base age of 50 years.
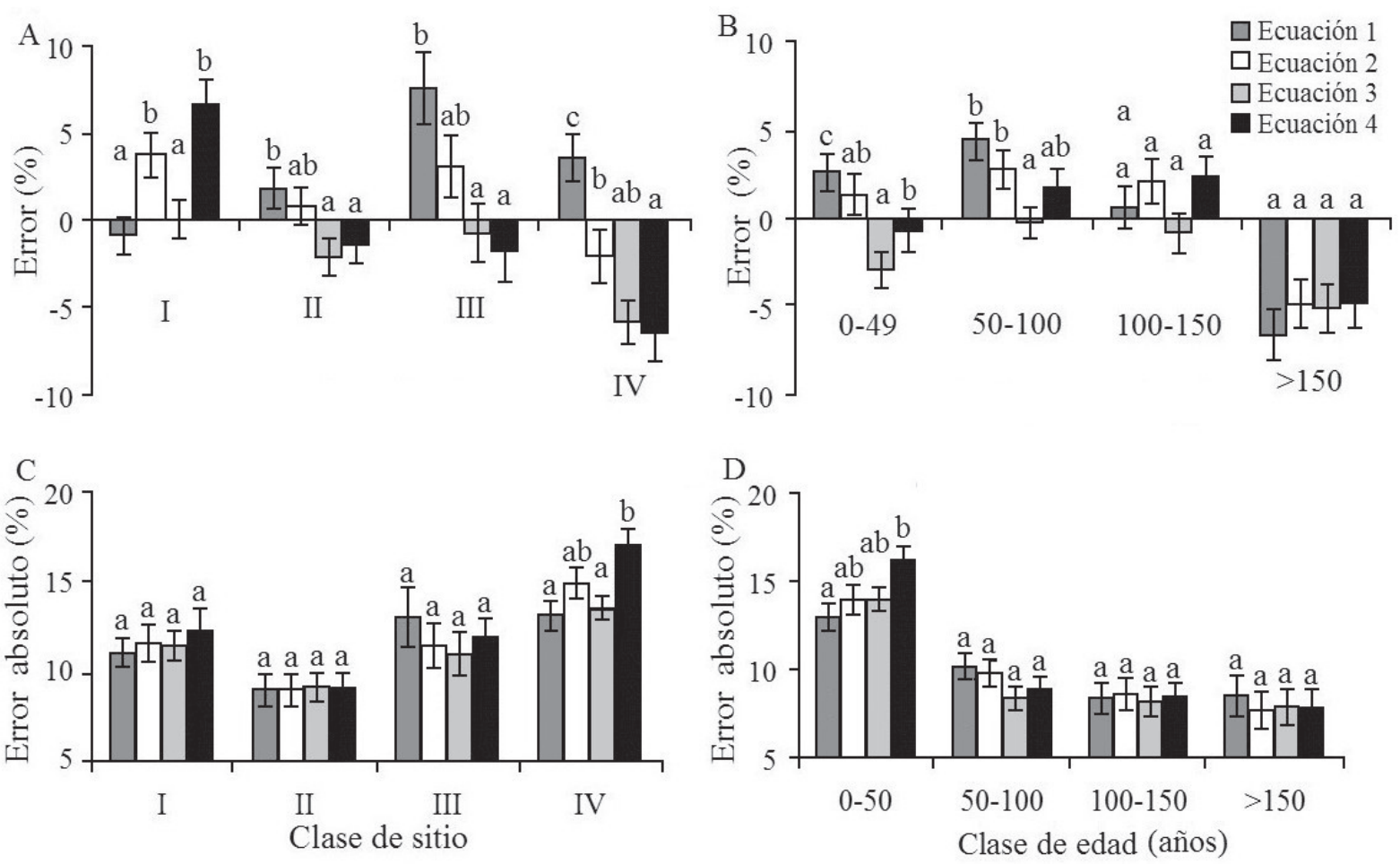

Figura 2. Errores medios porcentuales de la estimación (EME) por modelo de índice de sitio discriminado por clases de sitio (A) y clases de edad (B) y errores medios porcentuales en valor absoluto (\%|ê|) por modelo de índice de sitio discriminado por clases de sitio (C) y clases de edad (D) para una edad base de 50 años. Letras diferentes indican diferencias significativas a $P<0,05$ entre ecuaciones dentro de cada clase de sitio y edad.

Mean errors of estimation (EME) classified by model and site (A) and age (B) classes and mean absolute percentage error (\%|e||) by model classified by site (C) and age (D) classes at a base age of 50 years at a base age of 50 years. Different letters showed significant differences $(P<0.05)$ among equations within each site and age classes. 
ciones para las clases de sitio superiores, pero en la clase de sitio IV (figura 2C) de la ecuación [4] fue significativamente mayor al de las ecuaciones [1] y [3]. Mientras que la ecuación [2] no se diferenció significativamente del resto de las ecuaciones. Para todas las ecuaciones, los menores errores medios porcentuales en valor absoluto se encontraron en la clase II $(9,02,9,00,9,19$ y 9,17 para las ecuaciones [1] a [4], respectivamente), mientras que los mayores valores se observaron en la clase IV $(13,16,14,97$, 13,60 y 17,04 para las ecuaciones [1] a [4], respectivamente). Los mayores errores medios porcentuales en valor absoluto se encontraron en la clase de edad más joven $(<50$ años) para todas las ecuaciones (figura 2D), disminuyendo progresivamente hacia la clase de edad superior. En la clase de edad inferior la ecuación [4] presentó un error medio porcentual en valor absoluto $(16,7 \%)$ significativamente mayor al de la ecuación [1] (13,0 \%), mientras que para el resto de las clases no hubo diferencias significativas con valores entre $10,2 \%$ y $8,4 \%$ para la segunda clase de edad (50-100 años), entre 8,7 \% y 8,2 \% para la tercer clase de edad (100-150 años) y entre 8,6 \% y 7,7 \% para la clase de edad superior ( $>150$ años).

En las curvas de crecimiento acumulado (figura 3) se puede observar que el crecimiento en altura es mayor durante los primeros 70 años para una clase de sitio I $\left(\mathrm{IS}_{50}>\right.$ $9,3 \mathrm{~m}$ ), y va retrasándose paulatinamente hacia la clase de sitio V $\left(\mathrm{IS}_{50}\right.$ entre 2,5 y $\left.3,6 \mathrm{~m}\right)$. Al llegar a edades avanzadas, los árboles ya alcanzaron una altura máxima y no modifican la altura total. Este comportamiento se observó en las mayores clases de sitio de todas las ecuaciones analizadas, pero solo se cumplió en las clases de sitio inferior para las ecuaciones [2] y [4] (figuras 3B y 3D), mientras que en las ecuaciones [1] y [3] las curvas no alcanzaron a formar una asíntota. Por otro lado, en las ecuaciones [2] y [4] se observó para el menor índice de sitio (IS = 3,6 m) que durante los primeros años casi no hubo crecimiento en altura.

El incremento medio anual fue superior en las mejores clases de sitio para todas las ecuaciones durante los primeros 70 años aproximadamente (figura 4). A partir de los 70 años la ecuación no forzada de Weibull [1] y la ecuación forzada de Goelz y Burk [3] mostraron menores incrementos medios anuales en las clases de sitio superiores comparados con las clases inferiores (figuras 4A y 4C). Por otra parte, la ecuación no forzada de Richards [2] y la ecuación forzada de Newnham [4] presentaron niveles similares de incremento medio anual para todas las clases de sitio una vez alcanzada la edad de 70 años (figuras 4B y 4D). En las ecuaciones [2] y [4], el incremento medio anual máximo se produce primero en la mejor calidad de sitio (IS $=9,3 \mathrm{~m}$ ) retrasándose dicho máximo hacia edades más tardías en las clases de sitio inferiores. Este comportamiento no fue observado en las ecuaciones [1] y [3].

Al analizar los errores puntuales que producen los modelos a la edad de 50 años, se observó que la ecuación [1] presentó desviaciones de $0,81 \mathrm{~m}$ para un índice de si-
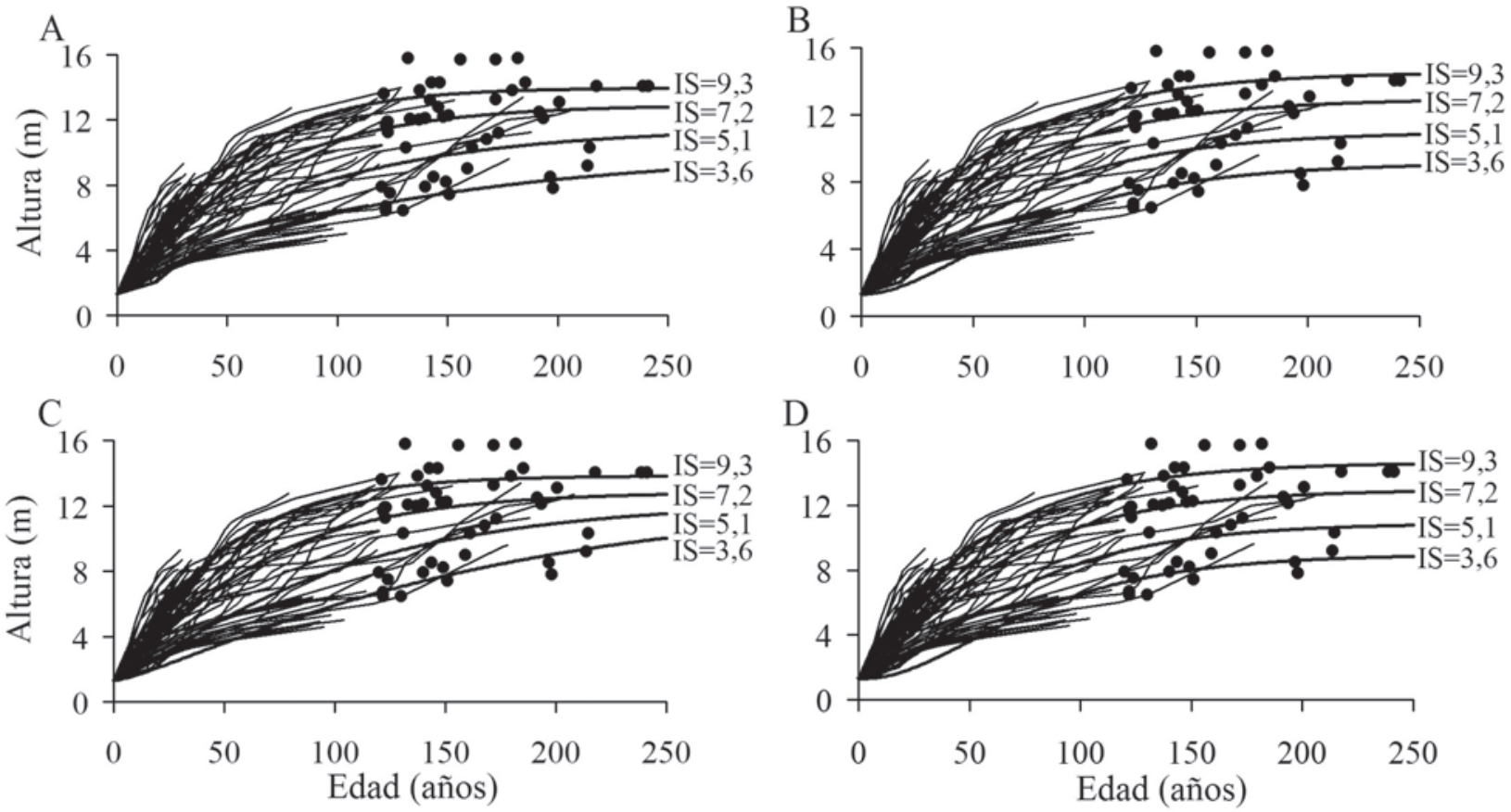

Figura 3. Conjunto de datos y curvas de crecimiento acumulado en altura. A: modelo de Weibull; B: modelo de Richards; C: modelo forzado Goelz-Burk; D: modelo forzado de Newnham; IS: índice de sitio.

Data set and height growth curves. A: Weibull model; B: Richards model; C: Goelz-Burk constrained model; D: Newnham constrained model; IS: site index. 

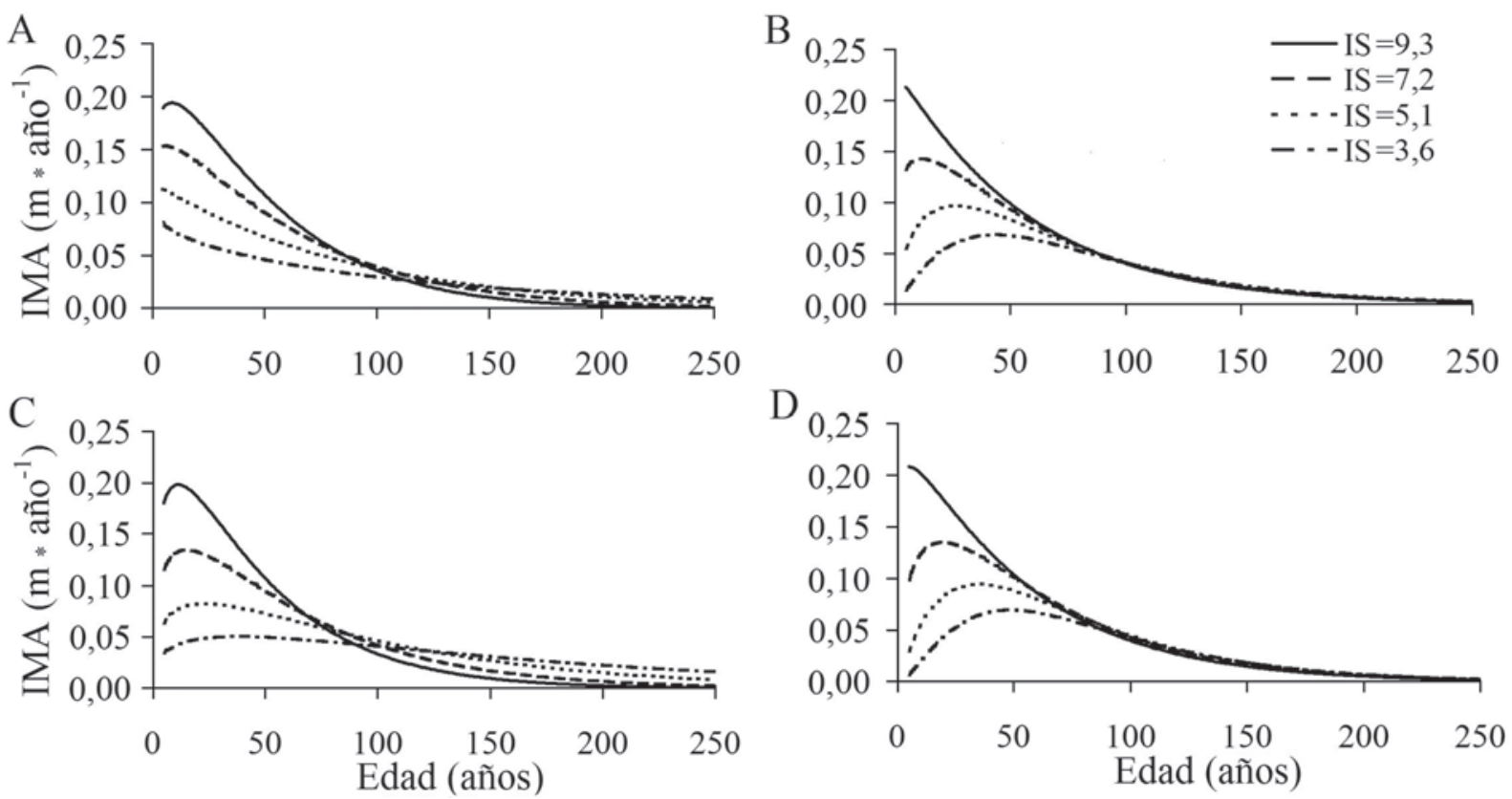

Figura 4. Curvas de incremento medio anual en altura (IMA). A: modelo de Weibull; B: modelo de Richards; C: modelo forzado Goelz-Burk; D: modelo forzado de Newnham; IS: índice de sitio.

Curves of mean annual increment. A: Weibull model; B: Richards model; C: Goelz-Burk constrained model; D: Newnham constrained model; IS: site index.

tio de 3,6 $\mathrm{m}$, de $0,77 \mathrm{~m}$ para un índice de sitio de $5,1 \mathrm{~m}$, $0,35 \mathrm{~m}$ para un índice de sitio de $7,2 \mathrm{~m}$ y $0,07 \mathrm{~m}$ para un índice de sitio de $9,3 \mathrm{~m}$. La ecuación [2], para los mismos índices de sitio, presentó desvíos de $0,23 \mathrm{~m}, 0,40 \mathrm{~m}$, $0,26 \mathrm{~m}$ y $0,20 \mathrm{~m}$, respectivamente. Mientras que las ecuaciones [3] y [4] al ser forzadas no presentaron desvíos a la edad de 50 años.

De las cuatro ecuaciones ensayadas, se seleccionó la ecuación forzada de Newnham [4] para la construcción de una clasificación de clases de sitio. De las otras funciones, la ecuación de Goelz y Burk se descartó por presentar un error medio de la estimación y un error medio porcentual en valor absoluto global muy elevados (cuadro 4). La ecuación [1] se descartó debido a la los errores puntuales que presentó a los 50 años de edad. La ecuación [2], si bien presenta estadísticos comparables a los de las ecuaciones [1] y [4] y las formas de las curvas son similares a las de la ecuación [4], no fue considerado debido a presentar errores puntuales a la edad de 50 que no están presentes en la ecuación [4].

Utilizando la ecuación de Newnham [4], quedó definida la siguiente clasificación de clases de sitio (CS) según el IS ${ }_{50}$ y la altura dominante (HD) a la que arribará un rodal a una edad mayor a los 150 años:

- CS I: $\mathrm{IS}_{50}>9,3 \mathrm{~m} ; \mathrm{HD}>14,0 \mathrm{~m}$

- CS II: IS I0 $_{5,2}$ - 9,3 m; HD 12,0 - 14,0 m

- CS III: IS $_{50} 5,1$ - 7,2 m; HD 10,0 - 11,9 m

- CS IV: $\mathrm{IS}_{50} 3,6$ - 5,1 m; HD 8,0 - 9,9 m

- CS V: $\mathrm{IS}_{50}<3,6 \mathrm{~m} ; \mathrm{HD}<8,0 \mathrm{~m}$

\section{DISCUSIÓN}

Los valores provenientes de la autovalidación de las ecuaciones seleccionadas son comparables a los encontrados por otros autores que trabajaron con curvas de índice de sitio aplicadas a bosques de Nothofagus spp. Los coeficientes de determinación ( $\mathrm{R}^{2}$-ajust.) calculados en este trabajo son similares al determinado por Martínez Pastur et al. (1997) quienes determinaron un $\mathrm{R}^{2}$-ajust de 94,4 para la función de índice de sitio forzada de Weibull de tres parámetros con datos de $N$. pumilio.

Como fue presentado en los resultados, dentro de una misma edad base los modelos de 4 y 5 parámetros muestran un $\mathrm{R}^{2}$-ajust. levemente mayores que los modelos generados con la ecuación de tres parámetros [4]. A su vez, se obtienen mayores valores de $\mathrm{R}^{2}$-ajust. en las ecuaciones no forzadas con respecto a las forzadas, que puede estar relacionado a una mayor plasticidad de estas ecuaciones. Los valores del coeficiente de Durbin-Watson indican que las ecuaciones para las edades base de 30 y 40 años no presentarían correlación de errores, mientras que en los casos en que se uso la edad base de 50 años posiblemente exista dicha correlación.

Las autovalidaciones de los modelos pueden conducir a conclusiones erróneas, dado que los supuestos de los modelos estadísticos no son confirmados con una muestra independiente. No obstante la autovalidación brinda una primera impresión de cuan efectivo puede ser un modelo a lo lago de diferentes gradientes analizados. Sin embargo, es recomendable, en las aplicaciones realizadas por poten- 
ciales usuarios, la realización de validaciones independientes para confirmar el ajuste de las estimaciones.

Los errores medios de la estimación obtenidos en este trabajo, para todas las ecuaciones excepto la ecuación [3], son similares a los encontrados para $N$. pumilio $(0,007 \mathrm{~m})$ (Martínez Pastur et al. 1997). La obtención de bajos valores de error medio de la estimación es debida a que los errores positivos se compensan con los negativos, dando, para las ecuaciones [1], [2] y [4], un promedio cercano a cero. Por otro lado, los valores de ESE son menores a los calculados por Martínez Pastur et al. (1997) (1,64 m), y levemente mayores a los calculados para $N$. obliqua y $N$. alpina (0,52 m y $0,39 \mathrm{~m}$, respectivamente) por Trincado et al. (2002) que generaron curvas polimórficas con una edad base de 20 años utilizando la ecuación de Cieszewski-Bella. Los errores medios porcentuales en valor absoluto (\%|ê|) calculados en este trabajo son levemente mayores a los calculados por Esse et al. (2007) a partir de la ecuación de índice de sitio de Chapman-Richards, con una edad base de 40 años para bosques de $N$. dombeyi.

El uso de ecuaciones forzadas evita la ocurrencia de errores ambiguos, por cuanto la altura predicha a la edad base es siempre igual a la indicada en el índice de sitio. Tal condición impone un forzamiento artificial sobre la ecuación, lo que resulta por lo general en un menor ajuste (Curtis et al. 1974, Martínez Pastur et al. 1997, Salas y García 2006). Sin embargo, las ecuaciones ajustadas presentan una mayor lógica en los datos, ya que dichos valores deben ser coincidentes. Por su parte, las ecuaciones no forzadas suelen tener mejores ajustes estadísticos, pero no cumplen con la condición de que la altura a la edad base sea igual al índice de sitio según la definición. Esto puede verse en el presente trabajo al observar que las ecuaciones no forzadas, presentan mejores ajustes globales que las ecuaciones forzadas (cuadro 4).

La utilización de una edad de referencia de 50 años permite obtener mejores ajustes globales que con las demás edades (30 y 40 años). Esto es consistente con lo encontrado por otros autores que determinaron que la elección de una edad de referencia cercana a la mitad del turno de corta produce menores errores y crea una distribución mas balanceada de los residuos (Gatzojannis 2000, Mamo y Sterba 2006). Según Teshome y Petty (2000) la elección de una edad base muy baja dará resultados severamente sesgados. Según lo citado por Ortega y Montero (1988) la determinación precisa de la edad base es clave porque de lo contrario puede producirse una clasificación errónea de las calidades de sitio. En el presente caso, la mayor edad ensayada resulta la más adecuada, no pudiendo determinarse si una edad mayor podría arrojar mejores ajustes.

Muchas veces un modelo puede presentar un muy buen comportamiento general, pero uno muy malo en forma particular, sobre todo en los extremos de los rangos considerados (Lencinas et al. 2002). Debido a esto es importante analizar el ajuste de los modelos a lo largo de gradientes de edad y de calidad de sitio, como los en- sayados en el presente trabajo. A partir del análisis de la distribución del error medio de la estimación no se puede establecer una clara diferenciación entre el ajuste de las ecuaciones forzadas y las no forzadas a lo largo de los gradientes considerados. Sin embargo, las distribuciones más balanceadas del error medio de la estimación a lo largo de dichos gradientes se obtienen con las ecuaciones [2] y [4]. La distribución del error medio porcentual en valor absoluto es similar para todos los modelos; sin embargo, los elevados valores de este parámetro para la menor clase de edad posiblemente se deban a que a edades tempranas, el crecimiento en altura de un rodal tenga una mayor influencia de otras variables externas a la calidad de sitio en comparación con el crecimiento en altura a edades más avanzadas (Nigh 1995). Por otra parte, cabe considerar que más importante que los valores alcanzados por los estadísticos lo es la buena significancia biológica de los modelos (Martínez Pastur et al. 2008). A partir de las curvas de crecimiento acumulado (figura 3 ) y de incremento medio anual (figura 4) es posible observar que las ecuaciones de Richards [2] y Newnham [4] cumplen con las condiciones que deben tener las curvas de crecimiento, o sea, una pendiente positiva, un punto de inflexión y alcanzar una asíntota que sea paralela al eje de las abscisas (Peschel 1938). Este comportamiento está definido por la genética de la especie y por las condiciones ambientales donde crece (Klepac 1983). En el caso de $N$. antarctica la forma de las curvas fue concordante con Donoso (2006), quien determina que estos bosques comienzan a mostrar signos de senescencia entre los 80 y los 120 años de edad. Las ecuaciones [1] y [3] no muestran un comportamiento armónico propio de esta descripción; por un lado, la ecuación [1] no presenta puntos de inflexión bien definidos para todas las clases de sitio, mientras que en la ecuación [3] las clases de sitio inferiores alcanzan dicho punto al mismo tiempo o antes que las clases superiores. Por otro lado, las ecuaciones [2] y [4] presentan la desventaja para la calidad de sitio inferior de poseer una curva que durante los primeros años casi no muestra crecimiento en altura.

Considerando los resultados analizados, las ecuaciones [1], [2] y [4] muestran los mejores ajustes, tanto en forma particular como general, sin presentar diferencias importantes entre ellos, pero si con la ecuación [3]. La superioridad las ecuaciones [2] y [4] también se observa en las características ya mencionadas de las curvas de crecimiento acumuladas y de incremento medio anual. No obstante, las ecuaciones [1] y [2], al ser no forzadas, presentan la desventaja de producir desviaciones, en mayor o menor grado, en la predicción de la altura a la edad base, mientras que la ecuación [4], debido a su condición de forzada, no produce dichas desviaciones. Con base en esto último se considera a la ecuación forzada de Newnham [4] como la más apropiada para los cálculos de índice de sitio en bosques de $N$. antarctica.

A partir del modelo obtenido, se puede observar que $N$. antarctica presenta un crecimiento en altura menor 
al de otras especies del género Nothofagus. Nothofagus dombeyi puede alcanzar, en los mejores sitios, alturas dominantes de $40 \mathrm{~m}$ a una edad de 80 años (Esse et al. 2007). Nothofagus pumilio, para la mejor calidad de sitio, alcanza los $30 \mathrm{~m}$ de altura a los 115 años de edad (Martínez Pastur et al. 1997). Nothofagus obliqua, en la mejor calidad de sitio, alcanza alturas cercanas a los 45 metros a una edad de 100 años (Salas y García 2006). Mientras que $N$. antarctica alcanza para la mejor calidad de sitio una altura dominante de $14 \mathrm{~m}$ a una edad de 150 años. Por otro lado, el incremento medio anual máximo en $N$. dombeyi, $N$. pumilio y $N$ obliqua, para las mejores calidades de sitio, es siempre superior al valor máximo de $N$. antarctica. En las mejores calidades de sitio, $N$. dombeyi, $N$. pumilio y $N$. obliqua presentan incrementos medios anuales máximos, respectivamente, de 0,67, 0,45 y 1,37 $\mathrm{m}_{\text {año }}{ }^{-1}$ (Martínez Pastur et al. 1997, Salas y García 2006, Esse et al. 2007), mientras que $N$. antarctica presenta un máximo de $0,23 \mathrm{~m}_{\text {año }} \mathrm{o}^{-1}$ para la mejor calidad de sitio.

La nueva clasificación de calidad de sitio propuesta, a partir de la ecuación [4] resulta una herramienta práctica para la estimación de la calidad de sitio. Teniendo en cuenta que el crecimiento en altura se estabiliza a una edad aproximada de 150 años (figura 3), el uso de dicha clasificación permite estimar la clase de sitio de un rodal adulto (> 150 años) con solo medir la altura dominante. Para rodales más jóvenes será necesario medir la altura dominante y la edad del rodal. Esta clasificación representa una alternativa a la anterior clasificación de clases de sitio presentada para $N$. antarctica por Lencinas et al. (2002).

\section{CONCLUSIONES}

El ajuste de una ecuación, en términos de calidad y comportamiento biológico, no puede ser determinado a priori para una especie en particular, y dependerán de la calidad de los datos obtenidos para el ajuste. En el presente caso, la condición de ecuaciones forzadas o no forzadas, así como la cantidad de parámetros no influyó significativamente. Si bien las ecuaciones forzadas $\mathrm{y}$ con menos parámetros presentaron ajustes levemente menores, la magnitud de esta diferencia no impidió que la ecuación forzada de Newnham de tres parámetros sea una herramienta práctica y precisa para estimar la altura de un rodal a partir de su edad e $\mathrm{IS}_{50}$. Se considera más importante en la implementación de un modelo de crecimiento, que éste tenga un adecuado comportamiento biológico y no produzca errores ambiguos a que se obtenga un menor ajuste estadístico dentro de ciertos limites. Es por ello que se recomienda el uso de la ecuación forzada de Newnham para ser usada como una herramienta precisa de predicción en la planificación del manejo de los bosques de N. antarctica.

\section{REFERENCIAS}

Clutter JL, JC Fortson, LV Pienaar, GH Brister, RL Bailey. 1983. Timber Management: A Cuantitative Approach. New York. John Wiley. 333 p.

Curtis RO, DJ DeMars, FR Herman. 1974. Which dependent variable in site index-height-age regression? Forest Science 20: 74-87.

Donoso C. 2006. Las especies arbóreas de los bosques Templados de Chile y Argentina. Autoecología. 3rd ed. Valdivia, Chile. Marisa Cuneo Ediciones. 678 p.

Ek A. 1971. A formula for white spruce site index curves. University Wisconsin Forestry Research Notes $N^{\circ} 161,2$ p.

Esse CR, CO Navarro, JC Pinares. 2007. Site index curves for Nothofagus dombeyi in the pre-Andean mountains of the province of Cautín, IX Region, Chile. Bosque 28(2): 142151.

Fekedulegn D, MP Mac Siurtain, JJ Colbert, 1999. Parameter estimation of nonlinear growth models in forestry. Silva Fennica 33(4): 327-336.

García O. 1983. A stochastic differential equation model for the height growth of forest stands. Biometrics 39:1059-1072.

Gatzojannis S. 2000. Site-index model for Abies chephalonica in the Mount Taygetos forests in Greece. Scandinavian Journal of Forest Research 15: 326-333.

Goelz JCG, TE Burk. 1992. Development of a well-behaved site index equation: Jack pine in north central Ontario. Canadian Journal of Forest Research 22: 776-784.

Gregoire T. 1993. Estimation of forest growth from successive surveys. Forest Ecology and Management 56: 267-278.

Gezan SA, PC Moreno M, A Ortega, 2009. Modelos fustales para renovales de roble, raulí y coigüe en Chile. Bosque 30(2): 61-69.

Khamis A, I Zuhaimy, H Khalid, 2005. Nonlinear growth models for modeling oil palm yield growth. Journal of Mathematics and Statistics 1(3): 225-233.

Klepac D. 1983. Crecimiento e incremento de árboles y masas forestales. Segunda edición. Universidad Autónoma Chapingo. Chapingo, México. 365 p.

Lei YC, SY Zhang, 2004. Features and partial derivatives of Bertalanffy-Richards growth model in forestry. Nonlinear Analysis: Modelling and Control 9(1): 65-73.

Lencinas MV, G Martínez Pastur, JM Cellini, R Vukasovic, PL Peri, C Fernández. 2002. Incorporación de la altura dominante y la calidad de sitio a ecuaciones estándar de volumen para Nothofagus antarctica (Forster f.) Oersted. Bosque 23(2): 5-17.

Mamo N, H Sterba. 2006. Site index functions for Cupressus lusitanica at Munesa Shashemene, Ethiopia. Forest Ecology and Management 237: 429-435.

Martínez Pastur G, PL Peri, R Vukasovic, S Vaccaro, V Piriz Carrillo. 1997. Site index equation for Nothofagus pumilio Patagonian forest. Phyton 61(1/2): 55-60.

Martínez Pastur G, PL Peri, C Fernández, G Staffieri, D Rodriguez. 1999. Desarrollo de la regeneración a lo largo del ciclo del manejo forestal de un bosque de Nothofagus pumilio: 2. Incidencia del ramoneo de Lama guanicoe. Bosque 20(2): 47-53.

Martínez Pastur G, JM Cellini, MV Lencinas, PL Peri. 2008. Stand growth model using volume increment/basal area ratios. Journal of Forest Science 54: 102-108.

McDill M, R Amateis. 1992. Measuring forest site quality using 
the parameters of a dimensionally compatible height growth function. Forest Science 38(2): 409-429.

Monserud RA. 1984. Height growth and site index curves for inland Douglas-fir based on stem analysis data and forest habitat type. Forest Science 30: 943-965.

Newnham R. 1988. A modification of the Ek-Payandeh nonlinear regression model for site index curves. Canadian Journal of Forest Research 18: 115-120.

Nigh G. 1995. Variable growth models for lodgepole pine in the sub-boreal spruce biogeoclimatic zone. British Columbia. Research Report RR-02. 22 p.

Ortega A, G Montero. 1988. Evaluación de la calidad de las estaciones forestales. Revisión bibliográfica. ICONA, Madrid. Ecología 2: 155-184.

Payandeh B, Y Wang. 1994. Modified site index equations for major Canadian timber species. Forest Ecology and Management 64: 97-101.

Peschel W. 1938. Die mathematischen methoden zur herleitung der wachstumsgesetze von baum und bestand und die ergebnisse ihrer anwendung. Tharandter forstlicher Jahrbuch 89: 169-247.

Pulido F, B Díaz, G Martínez Pastur. 2000. Incidencia del ramoneo del guanaco (Lama guanicoe) sobre la regeneración de lenga (Nothofagus pumilio) en bosques de Tierra del Fuego, Argentina. Investigación Agraria: Sistemas y Recursos Forestales 9(2): 381-394.

Salas C, O García. 2006. Modelling height development of mature Nothofagus obliqua. Forest Ecology and Management 229: 1-6.

Skovsgaard JP, JK Vanclay. 2008. Forest site productivity: a review of the evolution of dendrometric concepts for evenaged stands. Forestry 81(1): 13-31.

Splechtna B. 2001. Height growth and site index models for pacific silver fir in southwestern British Columbia. British Columbia Journal of Ecosystems and Management 1: 1-14.

Sturtevant BR, SW Seagle. 2004. Comparing estimates of forest site quality in old second-growth oak forests. Forest Ecology and Management 191: 311-328.

Teshome T, JA Petty. 2000. Site index equation for Cupressus lusitanica stands in Munesa forest Ethiopia. Forest Ecology and Management 126: 339-347.

Thrower JS. 1989. Site quality evaluation using site index. Silviculture Institute of British Columbia. $11 \mathrm{p}$.

Thrower JS, JW Goudie. 1992. Development of height-age and site-index functions for even-aged interior Douglas-fir in British Columbia. British Columbia Ministry of Forests. Research Note $\mathrm{N}^{\circ} 109.22$ p.

Trincado G, A Kiviste, K Von Gadow. 2002. Preliminary site index models for native roble (Nothofagus obliqua) and raulí (N. alpina) in Chile. New Zealand Journal of Forest Science 32(3): 322-333.

Tuhkanen S, I Kuokka, J Hyvonen, S Stenroos, J Niemela. 1989-1990. Tierra del fuego as a target for biogeographical research in the past and present. Anales del Instituto de la Patagonia 19(2): 5 -107.

Upadhyay A, T Eid, P Sankhayan. 2005. Construction of site index equations for even aged stands of Tectona grandis (teak) from permanent plot data in India. Forest Ecology and Management 212: 14-22.

Wang Y, B Payandeh. 1994a. A bi-segmental curve fitting approach to improve the accuracy of site index equations. Forest Ecology and Management 67: 35-38.

Wang Y, B Payandeh. 1994b. Application of the Kalman filter model in site index equation construction. Canadian Journal of Forest Research 24: 1415-1418.

Waring RH, KS Milner, WM Jolly, L Phillips, D McWethy. 2006. Assessment of site index and forest growth capacity across the Pacific and Inland Northwest U.S.A. with MODIS satellite-derived vegetation index. Forest Ecology and Management 228: 285-291.

Zeide B. 1993. Analysis of growth equations. Forest Science 39(3): 594-616.

Recibido: 25.11.10

Aceptado: 11.04.11 
